Geometry \& Topology Monographs

Volume 3: Invitation to higher local fields

Part II, section 9, pages 293-298

\title{
9. Local reciprocity cycles
}

\author{
Ivan Fesenko
}

In this section we introduce a description of totally ramified Galois extensions of a local field with finite residue field (extensions have to satisfy certain arithmetical restrictions if they are infinite) in terms of subquotients of formal power series $\mathbb{F}_{p}^{\mathrm{sep}}[[X]]^{*}$. This description can be viewed as a non-commutative local reciprocity map (which is not in general a homomorphism but a cocycle) which directly describes the Galois group in terms of certain objects related to the ground field. Abelian class field theory as well as metabelian theory of Koch and de Shalit [K], [KdS] (see subsection 9.4) are partial cases of this theory.

\subsection{Group $U_{N(\widehat{L / F})}^{\diamond}$}

Let $F$ be a local field with finite residue field. Denote by $\varphi \in G_{F}$ a lifting of the Frobenius automorphism of $F_{\text {ur }} / F$.

Let $F^{\varphi}$ be the fixed field of $\varphi$. The extension $F^{\varphi} / F$ is totally ramified.

Lemma ([KdS, Lemma 0.2]). There is a unique norm compatible sequence of prime elements $\pi_{E}$ in finite subextensions $E / F$ of $F^{\varphi} / F$.

Proof. Uniqueness follows from abelian local class field theory, existence follows from the compactness of the group of units.

In what follows we fix $F^{\varphi}$ and consider Galois subextensions $L / F$ of $F^{\varphi} / F$. Assume that $L / F$ is arithmetically profinite, ie for every $x$ the ramification group $\operatorname{Gal}(L / F)^{x}$ is open in $\operatorname{Gal}(L / F)$ (see also subsection 6.3 of Part II). For instance, a totally ramified $p$-adic Lie extension is arithmetically profinite.

For an arithmetically profinite extension $L / F$ define its Hasse-Herbrand function $h_{L / F}:[0, \infty) \rightarrow[0, \infty)$ as $h_{L / F}(x)=\lim h_{M / F}(x)$ where $M / F$ runs over finite subextensions of $L / F$ (cf. [FV, Ch. III $\S 5]$ ). 
If $L / F$ is infinite let $N(L / F)$ be the field of norms of $L / F$. It can be identified with $k_{F}((\Pi))$ where $\Pi$ corresponds to the norm compatible sequence $\pi_{E}$ (see subsection 6.3 of Part II, [W], [FV, Ch.III §5]).

Denote by $\varphi$ the automorphism of $N(L / F)_{\mathrm{ur}}$ and of its completion $N \widehat{(L / F)}$ corresponding to the Frobenius automorphism of $F_{\mathrm{ur}} / F$.

Definition. Denote by $U_{N(\widehat{L / F})}$ the subgroup of the group $U_{N(\widehat{L / F})}$ of those elements whose $\widehat{F}$-component belongs to $U_{F}$. An element of $U_{N \widehat{(L / F)}}^{\diamond}$ such that its $\widehat{F}$-component is $\varepsilon \in U_{F}$ will be called a lifting of $\varepsilon$.

The group $U_{N(L / F)}^{\diamond} / U_{N(L / F)}$ is a direct product of a quotient group of the group of multiplicative representatives of the residue field $k_{F}$ of $F$, a cyclic group $\mathbb{Z} / p^{a}$ and a free topological $\mathbb{Z}_{p}$-module. The Galois group $\operatorname{Gal}(L / F)$ acts naturally on $U_{N(L / F)}^{\diamond} / U_{N(L / F)}$.

\subsection{Reciprocity map $\mathcal{N}_{L / F}$}

To motivate the next definition we interpret the map $\Upsilon_{L / F}$ (defined in 10.1 and 16.1) for a finite Galois totally ramified extension $L / F$ in the following way. Since in this case both $\pi_{\Sigma}$ and $\pi_{L}$ are prime elements of $L_{\mathrm{ur}}$, there is $\varepsilon \in U_{L_{\mathrm{ur}}}$ such that $\pi_{\Sigma}=\pi_{L} \varepsilon$. We can take $\tilde{\sigma}=\sigma \varphi$. Then $\pi_{L}^{\sigma-1}=\varepsilon^{1-\sigma \varphi}$. Let $\eta \in U_{\widehat{L}}$ be such that $\eta^{\varphi-1}=\varepsilon$. Since $\left(\eta^{\sigma \varphi-1} \varepsilon^{-1}\right)^{\varphi-1}=\left(\eta^{(\sigma-1) \varphi}\right)^{\varphi-1}$, we deduce that $\varepsilon=\eta^{\sigma \varphi-1} \eta^{(1-\sigma) \varphi} \rho$ with $\rho \in U_{L}$. Thus, for $\xi=\eta^{\sigma \varphi-1}$

$$
\Upsilon_{L / F}(\sigma) \equiv N_{\Sigma / F} \pi_{\Sigma} \equiv N_{\widehat{L} / \widehat{F}} \xi \bmod N_{L / F} L^{*}, \quad \xi^{1-\varphi}=\pi_{L}^{\sigma-1} .
$$

Definition. For a $\sigma \in \operatorname{Gal}(L / F)$ let $U_{\sigma} \in U_{\widehat{N(L / F)}}$ be a solution of the equation

$$
U^{1-\varphi}=\Pi^{\sigma-1}
$$

(recall that id $-\varphi: U_{\widehat{N(L / F)}} \rightarrow U_{N \widehat{(L / F)}}$ is surjective). Put

$$
\mathcal{N}_{L / F}: \operatorname{Gal}(L / F) \rightarrow U_{N(L / F)}^{\diamond} / U_{N(L / F)}, \quad \mathcal{N}_{L / F}(\sigma)=U_{\sigma} \quad \bmod U_{N(L / F)} .
$$

Remark. Compare the definition with Fontaine-Herr's complex defined in subsection 6.4 of Part II.

\section{Properties.}

(1) $\mathcal{N}_{L / F} \in Z^{1}\left(\operatorname{Gal}(L / F), U_{N(\widehat{L / F})}^{\diamond} / U_{N(L / F)}\right)$ is injective.

Geometry \& Topology Monographs, Volume 3 (2000) - Invitation to higher local fields 
(2) For a finite extension $L / F$ the $\widehat{F}$-component of $\mathcal{N}_{L / F}(\sigma)$ is equal to the value $\Upsilon_{L / F}(\sigma)$ of the abelian reciprocity map $\Upsilon_{L / F}$ (see the beginning of 9.2).

(3) Let $M / F$ be a Galois subextension of $L / F$ and $E / F$ be a finite subextension of $L / F$. Then the following diagrams of maps are commutative:

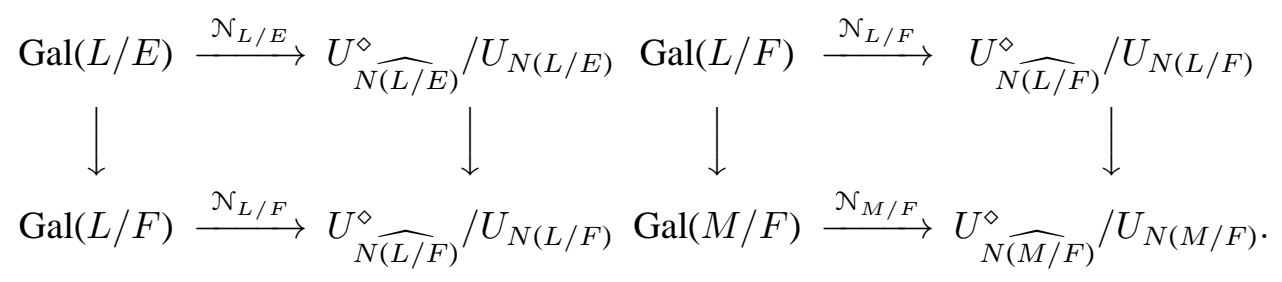

(4) Let $U_{n, N \widehat{(L / F)}}^{\diamond}$ be the filtration induced from the filtration $U_{n, N(\widehat{L / F})}$ on the field of norms. For an infinite arithmetically profinite extension $L / F$ with the HasseHerbrand function $h_{L / F}$ put $\operatorname{Gal}(L / F)_{n}=\operatorname{Gal}(L / F)^{h_{L / F}^{-1}(n)}$. Then $\mathcal{N}_{L / F}$ maps $\operatorname{Gal}(L / F)_{n} \backslash \operatorname{Gal}(L / F)_{n+1}$ into $U_{n, N(L / F)}^{\diamond} U_{N(L / F)} \backslash U_{n+1, N(L / F)}^{\diamond} U_{N(L / F)}$.

(6) The set $\operatorname{im}\left(\mathcal{N}_{L / F}\right)$ is not closed in general with respect to multiplication in the group

$U_{N \widehat{(L / F)}} / U_{N(L / F)}$. Endow $\operatorname{im}\left(\mathcal{N}_{L / F}\right)$ with a new group structure given by $x \star y=$ $x \mathcal{N}_{L / F}^{-1}(x)(y)$. Then clearly $\operatorname{im}\left(\mathcal{N}_{L / F}\right)$ is a group isomorphic to $\operatorname{Gal}(L / F)$.

Problem. What is $\operatorname{im}\left(\mathcal{N}_{L / F}\right)$ ?

One method to solve the problem is described below.

\subsection{Reciprocity map $\mathcal{H}_{L / F}$}

Definition. Fix a tower of subfields $F=E_{0}-E_{1}-E_{2}-\ldots$, such that $L=\cup E_{i}$, $E_{i} / F$ is a Galois extension, and $E_{i} / E_{i-1}$ is cyclic of prime degree. We can assume that $\left|E_{i+1}: E_{i}\right|=p$ for all $i \geqslant i_{0}$ and $\left|E_{i_{0}}: E_{0}\right|$ is relatively prime to $p$.

Let $\sigma_{i}$ be a generator of $\operatorname{Gal}\left(E_{i} / E_{i-1}\right)$. Denote

$$
X_{i}=U_{\widehat{E}_{i}}^{\sigma_{i}-1} .
$$

The group $X_{i}$ is a $\mathbb{Z}_{p}$-submodule of $U_{1, \widehat{E_{i}}}$. It is the direct sum of a cyclic torsion group of order $p^{n_{i}}, n_{i} \geqslant 0$, generated by, say, $\alpha_{i}\left(\alpha_{i}=1\right.$ if $\left.n_{i}=0\right)$ and a free topological $\mathbb{Z}_{p}$-module $Y_{i}$.

We shall need a sufficiently "nice" injective map from characteristic zero or $p$ to characteristic $p$

$$
f_{i}: U_{\widehat{E}_{i}}^{\sigma_{i}-1} \rightarrow U_{N\left(\widehat{L / E}_{i}\right)} \rightarrow U_{N(L / F)}
$$

Geometry \& Topology Monographs, Volume 3 (2000) - Invitation to higher local fields 
If $F$ is a local field of characteristic zero containing a non-trivial $p$ th root $\zeta$ and $f_{i}$ is a homomorphism, then $\zeta$ is doomed to go to 1 . Still, from certain injective maps (not homomorphisms) $f_{i}$ specifically defined below we can obtain a subgroup $\prod f_{i}\left(U_{\widehat{E}_{i}}^{\sigma_{i}-1}\right)$ of $U_{N(\widehat{L / F})}^{\diamond}$

Definition. If $n_{i}=0$, set $A^{(i)} \in U_{N\left(\widehat{L / E}_{i}\right)}$ to be equal to 1 .

If $n_{i}>0$, let $A^{(i)} \in U_{N\left(\widehat{L / E_{i}}\right)}$ be a lifting of $\alpha_{i}$ with the following restriction: $A_{\widehat{E_{i+1}}}^{(i)}$ is not a root of unity of order a power of $p$ (this condition can always be satisfied, since the kernel of the norm map is uncountable).

$\operatorname{Lemma}([\mathrm{F}])$. If $A^{(i)} \neq 1$, then $\beta_{i+1}=A_{E_{i+1}^{(i)}}^{p^{n_{i}}}$ belongs to $X_{i+1}$.

Note that every $\beta_{i+1}$ when it is defined doesn't belong to $X_{i+1}^{p}$. Indeed, otherwise we would have $A_{E_{i+1}}^{(i)} p^{n_{i}}=\gamma^{p}$ for some $\gamma \in X_{i+1}$ and then $A_{E_{i+1}}^{(i) p^{n_{i}-1}}=\gamma \zeta$ for a root $\zeta$ of order $p$ or 1 . Taking the norm down to $\widehat{E_{i}}$ we get $\alpha_{i}^{p^{n_{i}-1}}=N_{\widehat{E_{i+1}} / \widehat{E}_{i}} \gamma=1$, which contradicts the definition of $\alpha_{i}$.

Definition. Let $\beta_{i, j}, j \geqslant 1$ be free topological generators of $Y_{i}$ which include $\beta_{i}$ whenever $\beta_{i}$ is defined. Let $B^{(i, j)} \in U_{N \widehat{\left(L / E_{i}\right)}}$ be a lifting of $\beta_{i, j}$ (i.e. $B^{(i, j)} \widehat{E}_{i}=$ $\left.\beta_{i, j}\right)$, such that if $\beta_{i, j}=\beta_{i}$, then $B_{\widehat{E_{k}}}^{(i, j)}=B_{\widehat{E_{k}}}^{(i)}=A_{\mathrm{E}_{k}}^{(i-1)^{p^{n} i-1}}$ for $k \geqslant i$.

Define a map $X_{i} \rightarrow U_{N\left(\widehat{(L / E}_{i}\right)}$ by sending a convergent product $\alpha_{i}^{c} \prod_{j} \beta_{i, j}^{c_{j}}$, where $0 \leqslant c \leqslant n_{i}-1, c_{j} \in \mathbb{Z}_{p}$, to $A^{(i)^{c}} \prod_{j} B^{(i, j)^{c_{j}}}$ (the latter converges). Hence we get a map

$$
f_{i}: U_{\widehat{E}_{i}}^{\sigma_{i}-1} \rightarrow U_{N\left(\widehat{L / E}_{i}\right)} \rightarrow U_{N(L / F)}
$$

which depends on the choice of lifting. Note that $f_{i}(\alpha)_{\widehat{E}_{i}}=\alpha$.

Denote by $Z_{i}$ the image of $f_{i}$. Let

$$
\begin{aligned}
& Z_{L / F}=Z_{L / F}\left(\left\{E_{i}, f_{i}\right\}\right)=\left\{\prod_{i} z^{(i)}: z^{(i)} \in Z_{i}\right\}, \\
& Y_{L / F}=\left\{y \in U_{N \widehat{(L / F)}}: y^{1-\varphi} \in Z_{L / F}\right\} .
\end{aligned}
$$

Lemma. The product of $z^{(i)}$ in the definition of $Z_{L / F}$ converges. $Z_{L / F}$ is a subgroup of $U_{N(\widehat{L} / F)}$. The subgroup $Y_{L / F}$ contains $U_{N(L / F)}$.

Geometry \& Topology Monographs, Volume 3 (2000) - Invitation to higher local fields 
Theorem $([\mathrm{F}])$. For every $\left(u_{\widehat{E_{i}}}\right) \in U_{\widehat{N(L / F)}}^{\diamond}$ there is a unique automorphism $\tau$ in the group $\operatorname{Gal}(L / F)$ satisfying

$$
\left(u_{\widehat{E}_{i}}\right)^{1-\varphi} \equiv \Pi^{\tau-1} \bmod Z_{L / F} .
$$

If $\left(u_{\widehat{E}_{i}}\right) \in Y_{L / F}$, then $\tau=1$.

Hint. Step by step, passing from $\widehat{E_{i}}$ to $\widehat{E_{i+1}}$.

Remark. This theorem can be viewed as a non-commutative generalization for finite $k$ of exact sequence $(*)$ of 16.2 .

Corollary. Thus, there is map

$$
\mathcal{H}_{L / F}: U_{N(L / F)}^{\diamond} \rightarrow \operatorname{Gal}(L / F), \quad \mathcal{H}_{L / F}\left(\left(u_{\widehat{E_{i}}}\right)\right)=\tau .
$$

The composite of $\mathcal{N}_{L / F}$ and $\mathcal{H}_{L / F}$ is the identity map of $\operatorname{Gal}(L / F)$.

\subsection{Main Theorem}

Theorem ([F]). Put

$$
\mathcal{H}_{L / F}: U_{N(\widehat{L} / F)}^{\diamond} / Y_{L / F} \rightarrow \operatorname{Gal}(L / F), \quad \mathcal{H}_{L / F}\left(\left(u_{\widehat{E}}\right)\right)=\tau
$$

where $\tau$ is the unique automorphism satisfying $\left(u_{\widehat{E}}\right)^{1-\varphi} \equiv \Pi^{\tau-1} \bmod Z_{L / F}$. The injective map $\mathcal{H}_{L / F}$ is a bijection. The bijection

$$
\mathcal{N}_{L / F}: \operatorname{Gal}(L / F) \rightarrow U_{N(\mathcal{S} / F)}^{\diamond} / Y_{L / F}
$$

induced by $\mathcal{N}_{L / F}$ defined in 9.2 is a 1-cocycle.

Corollary. Denote by $q$ the cardinality of the residue field of $F$. Koch and de Shalit $[\mathrm{K}],[\mathrm{KdS}]$ constructed a sort of metabelian local class field theory which in particular describes totally ramified metabelian extensions of $F$ (the commutator group of the commutator group is trivial) in terms of the group

$$
\mathfrak{n}(F)=\left\{\left(u \in U_{F}, \xi(X) \in \mathbb{F}_{p}^{\text {sep }}[[X]]^{*}\right): \xi(X)^{\varphi-1}=\{u\}(X) / X\right\}
$$

with a certain group structure. Here $\{u\}(X)$ is the residue series in $\mathbb{F}_{p}^{\text {sep }}[[X]]^{*}$ of the endomorphism $[u](X) \in O_{F}[[X]]$ of the formal Lubin-Tate group corresponding to $\pi_{F}, q, u$.

Let $M / F$ be the maximal totally ramified metabelian subextension of $F_{\varphi}$, then $M / F$ is arithmetically profinite. Let $R / F$ be the maximal abelian subextension of $M / F$. Every coset of $U_{N \widehat{(M / F)}}^{\diamond}$ modulo $Y_{M / F}$ has a unique representative in 
$\operatorname{im}\left(\mathcal{N}_{M / F}\right)$. Send a coset with a representative $\left(u_{\widehat{Q}}\right) \in U_{N \widehat{(M / F)}}^{\diamond}(F \subset Q \subset M$, $|Q: F|<\infty)$ satisfying $\left(u_{\widehat{Q}}\right)^{1-\varphi}=\left(\pi_{Q}\right)^{\tau-1}$ with $\tau \in \operatorname{Gal}(M / F)$ to

$$
\left(u_{\widehat{F}}^{-1},\left(u_{\widehat{E}}\right) \in U_{N \widehat{(R / F)}}^{\diamond}\right) \quad(F \subset E \subset R,|E: F|<\infty) .
$$

It belongs to $\mathfrak{n}(F)$, so we get a map

$$
g: U_{N(\widehat{M} / F)}^{\diamond} / Y_{M / F} \rightarrow \mathfrak{n}(F) .
$$

This map is a bijection [F] which makes Koch-de Shalit's theory a corollary of the main results of this section.

\section{References}

[F] I. Fesenko, Nonabelian local reciprocity maps, to appear in Class Field Theory: Its Centenary and Prospect, ed. K. Miyake, Advanced Studies in Pure Mathematics, Math. Soc. Japan, Tokyo 2001.

[FV] I. Fesenko and S. Vostokov, Local Fields and Their Extensions, AMS, Providence, R.I., 1993.

[K] H. Koch, Local class field theory for metabelian extensions, In Proceed. 2nd Gauss Symposium. Conf. A: Mathematics and Theor. Physics (Munich, 1993), de Gruyter, Berlin, 1995, 287-300.

[KdS] H. Koch and E. de Shalit, Metabelian local class field theory, J. reine angew. Math. 478(1996), 85-106.

[W] J.-P. Wintenberger, Le corps des normes de certaines extensions infinies des corps locaux; applications, Ann. Sci. E.N.S., 4 série 16(1983), 59-89.

Department of Mathematics University of Nottingham

Nottingham NG7 2RD England

E-mail: ibf@maths.nott.ac.uk 